\title{
The effect of triangularity on fluctuations in a tokamak plasma
}

\author{
M. Fontana, L. Porte, S. Coda, O. Sauter and the TCV \\ team $\ddagger$ \\ Swiss Plasma Center, EPFL, Lausanne, 1015, Switzerland \\ E-mail: matteo.fontana@epf $1 . \mathrm{ch}$
}

May 2017

\begin{abstract}
The effects of plasma shaping, in particular triangularity $(\delta)$, on temperature fluctuations in the Tokamak à Configuration Variable (TCV) have been studied using the Correlation Electron Cyclotron Emission (CECE) system. It has been found that, in ohmic discharges with comparable density profiles, the relative fluctuation level measured at the edge is significantly reduced in plasmas with negative triangularity with respect to positive triangularity ones. Additionally, the critical temperature gradients for the onset of turbulence are observed to increase in the negative triangularity plasmas. An estimation of the correlation length of the fluctuating structure shows smaller structure size in the negative triangularity cases, which are known to be associated with improved confinement. Together, these observations suggest that changing triangularity from positive to negative strongly influences the nature of the turbulent fluctuations excited in the plasma.
\end{abstract}




\section{Introduction}

One of the long-standing problems in magnetic confinement fusion research is the fact that measured heat and energy fluxes are found to be at least one order of magnitude larger than those predicted by neoclassical theory. This is generally attributed to the interplay between multi-field microfluctuations such as those that can be generated by turbulent phenomena. A thorough study of how plasma characteristics influence these fluctuations is thus very important.

The Tokamak à Configuration Variable (TCV) [1] is a medium sized tokamak (major radius $\mathrm{R}=0.88 \mathrm{~m}$, minor radius $\mathrm{a}=0.25 \mathrm{~m}$ ) with up to $1.53 \mathrm{~T}$ magnetic field on axis and up to $1 \mathrm{MA}$ plasma current. Its main asset is the unparalleled shaping capability that allows the investigation of plasmas with elongation $0.9<\kappa<2.8$ and triangularity [2] $-0.7<\delta<1$.

These shaping capabilities have been exploited in past experiments, where it was found that one parameter strongly influencing electron heat transport is the plasma triangularity $(\delta)[3]$. It has in fact been observed that in L-mode discharges with similar density profiles, the same temperature profile can be achieved using half of the Electron Cyclotron Resonance Heating (ECRH) power in negative triangularity plasmas with respect to positive triangularity ones [4][5]. In discharges where the same heating power has been applied, the electron heat transport has been found to be strongly reduced from the edge to $\rho_{v o l}=0.4$ [5], where $\rho_{v o l}$ is defined as $\sqrt{V / V_{L C F S}}$ where $V_{L C F S}$ is the plasma volume included in the last closed flux surface.

Moreover this difference has been found to decrease for increasing effective collisionality, defined as the ratio between the electron-ion collision frequency and the curvature drift frequency. This can be approximated as $\nu_{e f f}=0.1 * R * Z_{e f f} * n_{e} / T_{e}^{2}[5]$ where $\mathrm{R}$ is the major plasma radius. Successive gyrokinetic simulations $[6][7][8]$ have shown that turbulence in these plasmas was mainly Trapped Electron Modes (TEM) dominated, and since collisionality acts as a stabilizing factor for this type of modes, it has been suggested that some change in the underlying turbulence characteristics may be tied to the shaping effect on fluctuations. This earlier works, however, only focused on macrospcopic quantities related to transport (electron heat flux, electron heat conductivity and electron energy confinement time) due to the lack of fluctuations measurements on TCV at the time $[3][4][5]$.

Despite the magnitude of $\delta$ (positive or negative) decreasing quickly when moving from the edge to the plasma interior, the observed confinement improvement extends well into the core $\left(\rho_{v o l}<0.4\right)[5]$. A coherent picture emerges based on a distinction between regions of different stiffness within the plasma. In this paper the same definition of stiffness used in [9] is considered: the existence of a critical temperature gradient, which cannot effectively be exceeded, in that increases in the heat flux cause an increase in the level of turbulence, instead of a further increase in the temperature inverse scale length $\left(R / L_{T_{e}}=R *\right.$ $\left.d \log \left(T_{e}\right) / d R\right)$. In TCV it has indeed been observed, particularly in L-mode, limited plasmas, that a region exists around the plasma mid-radius (tipically from the sawtooth inversion radius to $\rho_{v o l} \sim 0.8$ ), where the $T_{e}$ and $n_{e}$ scale lengths are generally insensitive to various plasma parameters, including edge triangularity [9]. This will be referred to in the following as the stiff region. For more external radial locations, instead, the profiles can be fit with a uniform gradient that is found to change when changing plasma conditions and thus this plasma region is considered to be nonstiff. Thus, even if the local flux-surface triangularity magnitude quickly decreases when moving towards the core, a localised confinement improvement up to the outer edge of the stiff region would be carried all the way across it by its constant logarithmic gradient.

Gyrokinetic simulations have also been employed to investigate the effects of shaping on turbulence. All these simulations were performed at the ion scale, hence excluding Electron Temperature Gradient moddes (ETG) and taking into accounts electromagnetic effects, which were found to be unimportant. Non-linear simulations run with the flux tube GS2 code [10], using the experimental profiles and equilibria of the ECRH heated experimental cases in [5], found that Trapped Electron Modes (TEM) are the dominant instability. Furthermore, they reproduced the heat flux variation at the plasma edge within error bars and found a partial stabilization of TEM in the negative triangularity case [6]. Other non-linear simulations performed using the GENE code [11], on the same experimental profiles and equilibria, again found TEM dominated fluctuations, reproduced the heat flux difference at the plasma edge and suggested that, to reproduce the penetration of the heat flux reduction in the plasma core, global simulations would be necessary. Moreover, indications were found that changing triangularity from positive to negative increases the critical electron temperature gradients for the saturated heat flux [7][8].

\section{New experiments}

\subsection{Profiles analysis}

In 2016, new experiments were performed where fluctuations diagnostics, in particular the Correlation Electron Cyclotron Emission (CECE), were available. CECE [12][13][14][15][16][17][18] is a technique that allows spatially localised measurements of low wave- 

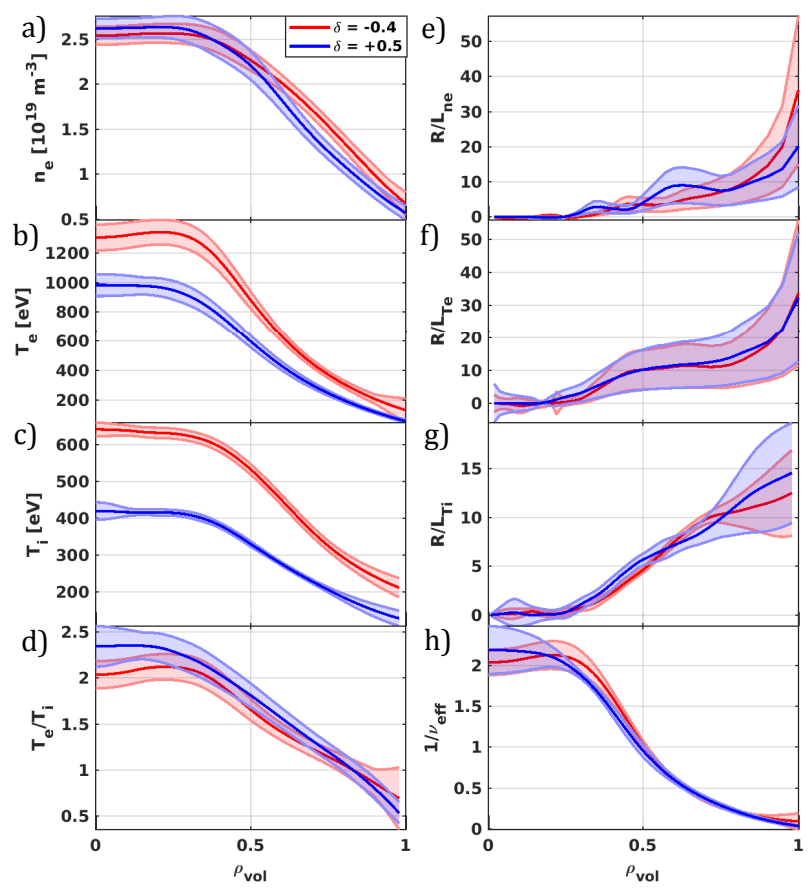

Figure 1: Profiles of the model discharges for the two most extreme shapes. a) Electron density. b) Electron temperature. c) Ion temperature. d)Electron/ion temperature ratio. e) Normalized density scalelength. f) Normalized electron temperature scale length. g) Normalized ion temperature scale length. h) Inverse effective collisionality. The electron profiles are calculated from Thomson scattering measurements while ion temperature and $Z_{\text {eff }}$ from charge exchange recombination spectroscopy measurements. Colors online.

number radiative temperature fluctuations. It exploits cross-correlation analysis on close but not overlapping plasma volumes to detect small fluctuations $(<1 \%)$. The CECE system of TCV [19] has recently been upgraded with a new Intermediate Frequency (IF) section containing six frequency tunable YIG filters with 100 $\mathrm{MHz}$ bandwidth granting very high $(<1 \mathrm{~cm})$ spatial resolution in the radial direction. This, combined with a steerable line of sight and the flexibility of TCV, allows the measurement of small $(<1 \%)$ electron temperature fluctuations with $k_{\theta}<1.12 \mathrm{~cm}^{-1}$ over a large fraction of the plasma cross-section. The signals are sampled at $1.75 \mathrm{MHz}$ and low-pass filtered at $450 \mathrm{kHz}$ before acquisition to improve the signal to noise ratio.

The effects of triangularity on the relative fluctuations amplitude were studied in ohmic discharges with $I_{p}=225 k A$, comparable density profiles with line averaged density of $210^{19} \mathrm{~m}^{-3}, \delta=$ $+0.5,+0.3,-0.3,-0.4$ and where the electron temperature was left free to evolve. The resulting profiles are shown in figure 1. Electron temperature and density profiles shown here are calculated from measurements from the Thomson scattering diagnostic [20], while ion profiles were obtained through the charge exchange recombination spectroscopy diagnostic of TCV [21]. Examples of the resulting plasma cross sections for the $\delta=+0.5$ and $\delta=-0.4$ are shown in figure 3 .

As expected, the negative triangularity discharges show a higher temperature over the whole profile. Note that this also implies that the ohmic power, calculated as the product of the plasma current and the voltage measured by the flux loops around the vacuum vessel in a stationary phase, actually decreases in going from positive to negative triangularity, from $329 \mathrm{~kW}$ at $\delta=+0.5$ to $287 \mathrm{~kW}$ at $\delta=-0.4$. Both effects therefore contribute to the confinement improvement. In particular, comparing the discharges with $\delta=+0.5$ with respect to those with $\delta=-0.4$, a central region where the temperature ratio is almost constant at $\sim 1.4$ can be distinguished between $0.45<$ $\rho_{\text {vol }}<0.7$ (figure 2). This is what is expected to happen in the stiff region of a plasma. A confirmation of this hypothesis can be found by looking at the normalized temperature gradients for the differently shaped discharges (figure 1f). In the same radial range, despite the differences in the temperature profiles, the value of the inverse temperature scale length is very similar across the different shapes considered. For $\rho_{v o l}>0.75$, instead, $R / L_{T e}$ changes and the temperature gradient of the negative $\delta$ discharges grows compared with the positive $\delta$ one. This is hence identified as the non-stiff region where the edge triangularity effectively exerts its influence. These discharges are ideal targets to observe how fluctuations change in positive and negative triangularity plasmas. Care was taken in selecting the line of sight and the CECE channels frequencies such to obtain fluctuations measurements both in the stiff and non-stiff regions of the plasma.

\subsection{Effects of optical depth}

It is important to stress that TCV plasmas are not optically thick over the whole volume. In fact, for the discharges considered here, the optical depth $\tau>2$ only for $\rho_{v o l}<0.55$. This implies that the measured fluctuations are a convolution of electron temperature and density fluctuations, where the relative weight of the latter increases with lower $\tau$ and strongly depends on the phase $\phi$ between $T_{e}$ and $n_{e}$ fluctuations. On TCV it is not yet possible to have measurements of density-temperature cross phase to precisely quantify the relative weight of these contributions. Nevertheless it is possible to estimate an upper boundary using the results of [22], considering the case in which density and temperature fluctuations are perfectly in phase. 


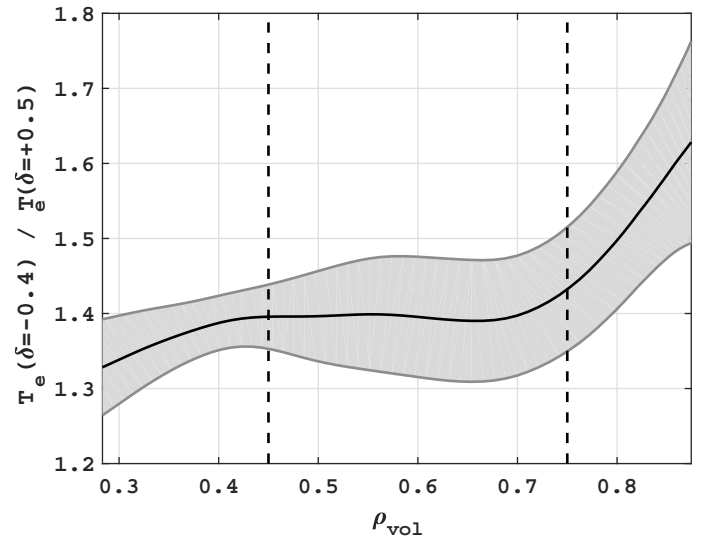

Figure 2: Electron temperature ratio between the $\delta=-0.4$ and $\delta=+0.5$ discarges.

In this case the measured radiative temperature $T_{\text {rad }}$ fluctuations would depend only on $R=\frac{\delta n_{e}}{n_{e}} / \frac{\delta T_{e}}{T_{e}}$, the ratio between temperature and density fluctuations, $\tau$ and the wall reflectivity $\chi$. The dependence from the last two parameters is summarised in the function $A 2(\tau, \chi)$ defined in the aforementioned [22]. Finally the measured radiative temperature fluctuations can be expressed as the sum of three components:

$$
\begin{aligned}
\left(\frac{\delta T_{\text {rad }}}{T_{\text {rad }}}\right)^{2}= & \left(\frac{\delta T_{e}}{T_{e}}\right)^{2}\left\{[1+A 2(\tau, \chi)]^{2}+\right. \\
& +A 2(\tau, \chi)^{2} R^{2}+ \\
& +2[1+A 2(\tau, \chi)] A 2(\tau, \chi) R\}
\end{aligned}
$$

where the first term represents the contribution of $T_{e}$ fluctuations exclusively, the second that of $n_{e}$, and the third is the result of the correlation of the two.

From the optical depth profiles for these experiments, $\tau \sim 0.5$ at $\rho_{\text {vol }}=0.85$, and a conservative value for the reflectivity of the carbon tiles (as in TCV) is $\chi \sim 0.76$ [23] resulting in $A 2(\tau, \chi)=0.33$. Preliminary non-linear, electromagnetic, gyrokinetic simulations suggest that the ratio $R$ should be between 0.5 and 1. The latter will be considered, in the frame of an upper limit estimation. The resulting maximum relative contribution of density on the measured fluctuation would then be between 10 and $15 \%$ at $\rho_{\text {vol }}=0.85$. For radial positions closer to the core this value quickly decreases. In any case, in this work, an exact estimation of the electron temperature fluctuations will not be attempted but only fluctuations of the radiative temperature will be discussed.

\subsection{Temperature fluctuations measurements}

After the development of a target for the four different shapes, each of them was repeated several times, with
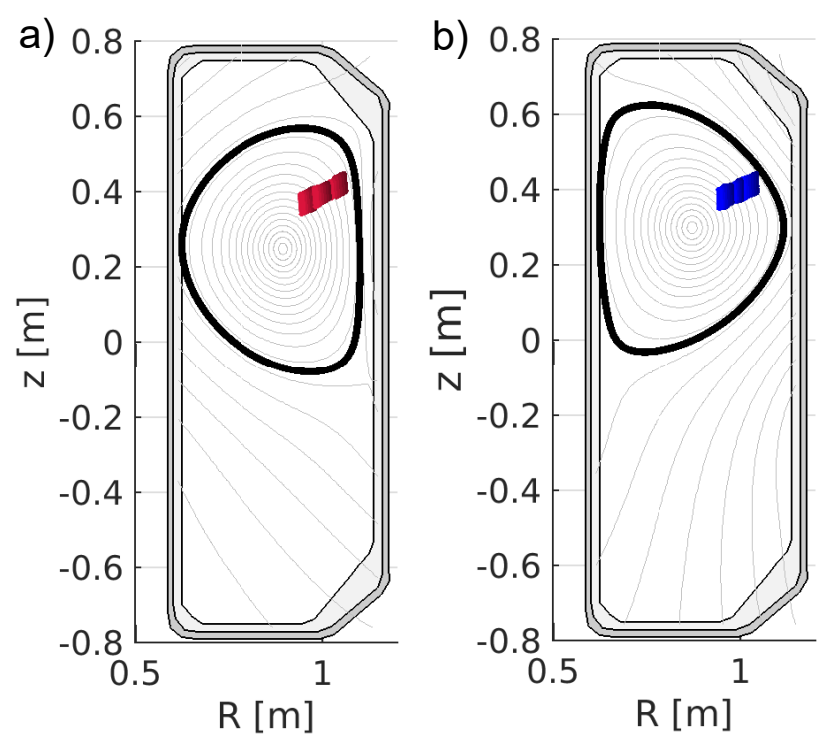

Figure 3: Plasma cross sections and position of the CECE emission volumes for the measurements taken with $\delta=-0.4$ (a) and $\delta=+0.5$ (b). Colors online.

different frequency settings for the CECE channels. In this way it was possible to obtain measurements from plasma volumes covering the region $0.3<\rho_{\text {vol }}<0.95$ and $0.3<\rho_{v o l}<0.85$ for the positive and negative triangularity discharges respectively. The position of the emission volume for each channel has been calculated via ray tracing, using the TORAY code [24] [25]. The repeated discharges showed excellent reproducibility. In figure 3 the shapes of the two target discharges for $\delta=+0.5$ and $\delta=-0.4$ are shown together with the reconstructed position of the emission volumes of the whole set of measurements. The chosen frequency spacing of the channels (500 $\mathrm{MHz}$ ) corresponds to physical distances varying from $0.8 \mathrm{~cm}$ for the outer channels to $0.65 \mathrm{~cm}$ for the inner ones, in any case shorter than the expected correlation lengths of fluctuating structures of interest. Crosscorrelation analysis was performed for each couple of adjacent channels, over a $100 \mathrm{~ms}$ time period. Examples of the spectra obtained with this technique are shown in figure 4 . In these plots it can be observed that positive triangularity discharges show a broadband feature between $\sim 20$ and $160 \mathrm{kHz}$, absent in the negative triangularity case. This feature seems to reduce in both amplitude and bandwidth moving towards the plasma core. The relative fluctuations amplitude between two channels is calculated from an integral of their cross power spectral density $G_{x y}$ :

$\frac{\delta T_{\text {rad }}}{T_{e}}=\frac{1}{T_{e}} \sqrt{\int_{f_{a}}^{f_{b}} G_{x y}(f) d f}$ 

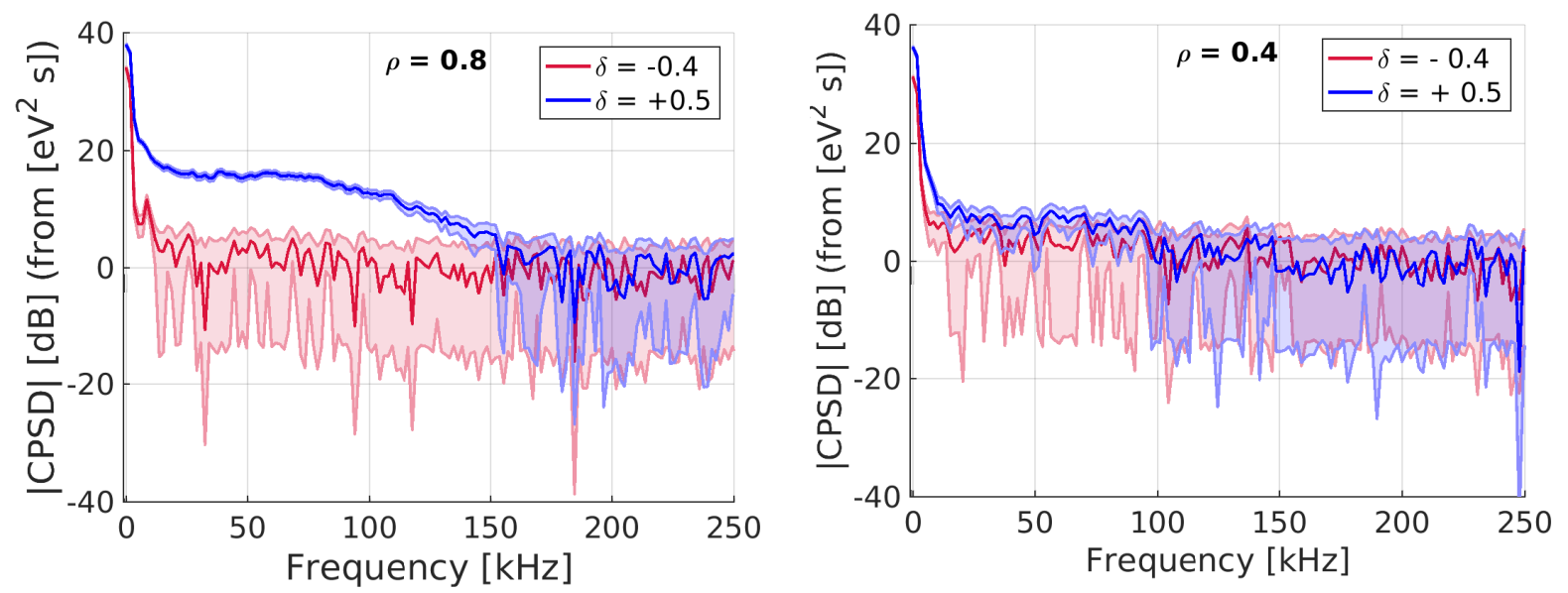

Figure 4: Cross Power Spectral Density obtained from correlation analysis over $100 \mathrm{~ms}$ of CECE signals coming from couples of neighbouring channels at $\rho_{v o l}=0.8 \rho_{v o l}=0.4$, in positive and negative triangularity. The noise level is at $0 \mathrm{~dB}$ for all the curves. Colors online.

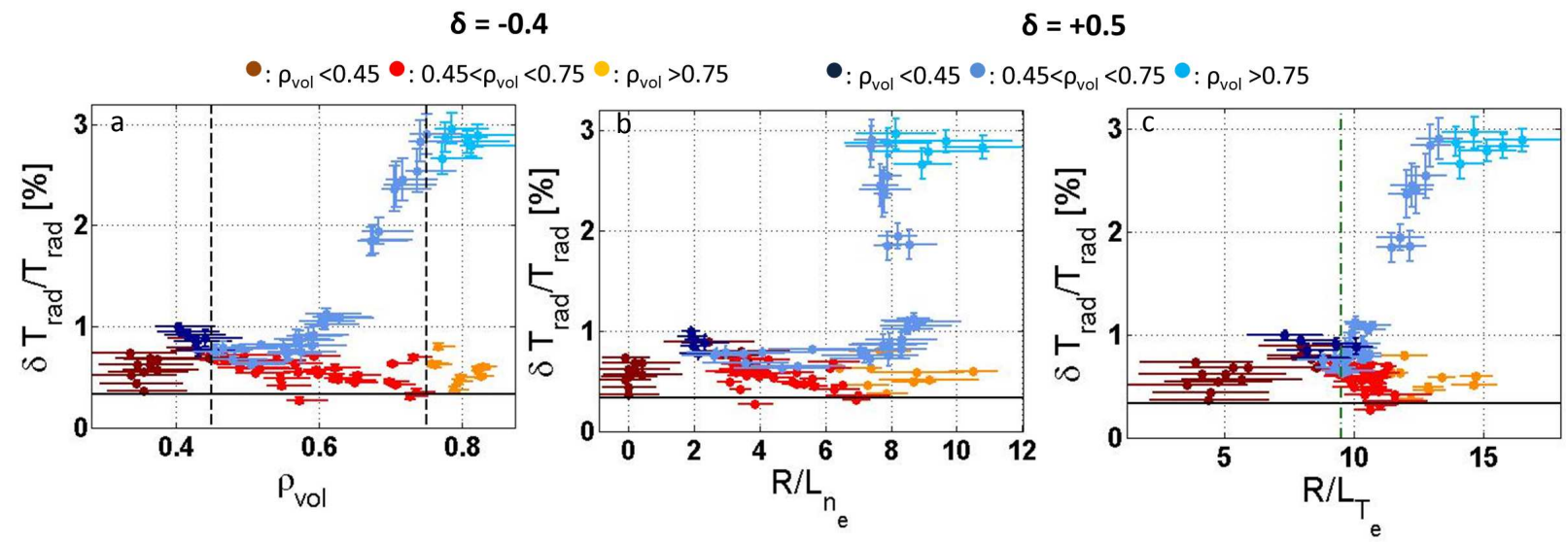

Figure 5: a)Radial profiles of relative radiative temperature fluctuations amplitude for different triangularity values. The fluctuations amplitude is reduced changing shape from positive to negative triangularity and the effects extends up to $\rho_{v o l}=0.5$. The same data are plotted against the normalized density (b) and temperature (c) scale lengths. Notice that these data points combine measurements taken over a wide fraction of the radial profile. Colors online.

In this formula $f_{a}$ and $f_{b}$ define the frequency range of the fluctuations whose amplitude is to be calculated. The spectra shown in figure 4 can be roughly divided in two parts. The high frequency part $(20-200 \mathrm{kHz})$ is where turbulent micro fluctuations are expected to be observed in TCV. The low frequency range (0$20 \mathrm{kHz}$ ) could still contain fluctuations, like zonal flows, that are known to influence the saturated turbulence amplitude [26], but it is normally not taken into account in TCV due to the strength of low frequency components (MHD modes, sawteeths). Furthermore negative triangularity is known to have an effect on sawteeth period and amplitude [27], so differences in this range cannot be directly associated with changes in the turbulence activity. In this case, anyway, negative and positive triangularity discharges showed comparable values of absolute fluctuations amplitude in the $0-20 \mathrm{kHz}$ spectrum, over the whole range of measurements. Taking into account the radiometer characteristics [12] and the averaging time, the minimum detectable fluctuation is:

$\delta T_{e} / T_{e}=\sqrt{\frac{2 B_{v i d}}{B_{I F}} \frac{1}{\sqrt{N_{s}}}}=\sim 0.45 \%$.

where $B_{v i d}$ is the signal bandwidth before acquisition, $B_{I F}$ the $3 \mathrm{~dB}$ bandwidth of the IF filters and $N_{s}$ the number of samples over which the correlation is being calculated.

The profiles of relative temperature fluctuations, $\delta T_{\text {rad }} / T_{e}$, shown in figure $5 \mathrm{a}$, clearly show a strong 
suppression of the fluctuations, especially close to the edge, in negative triangularity compared to positive triangularity. At $\rho_{v o l} \sim 0.8$ the fluctuation level in the $\delta=-0.4$ case is less than one third with respect to the one with $\delta=0.4$. The suppression of the fluctuations can also be observed in the absolute fluctuations amplitude (not shown here) proving that the difference in temperature is not the only factor responsible for this effect.

For radial positions closer to the plasma core the difference quickly decreases, but it is interesting to note that fluctuations in positive triangularity discharges remain measurably higher than those in negative triangularity down to $\rho_{v o l}=0.55$, where the magnitude of the plasma triangularity has already decreased to less than $\sim 40 \%$ its edge value.

These measurements, taken over a wide fraction of the radial profile, have also been used to study the dependence of the relative fluctuations on the normalized density and temperature scale lengths, as shown in figure $5 \mathrm{~b}$ and $\mathrm{c}$. Notice that, over the plasma region considered, these scale lengths are not the only parameters changing. Nonetheless, the plots suggest that a "critical" or threshold gradient is present in both temperature and density, corresponding to the point of sharp increase in fluctuation level. In the positive $\delta$ discharges, the sharp fluctuation rise occurs in a small region, corresponding to the stiff region of the plasma, where they increase until they reach the level measured at the edge. A similar trend is not observed in negative triangularity discharges. It could then be hypothesized that negative triangularity discharges have a higher critical gradient in terms of the behavior of fluctuations, beyond the range explored in these experiments. This is in agreement with the results of the non-linear gyrokinetic simulations performed in [7][8]. The exploration of a larger range of scale lengths and a more in depth study of the radial dependence of these critical gradients will be addressed in future work.

Cross-correlation analysis between the different CECE channels provides the means to estimate the radial correlation length $\left(L_{c}\right)$ of the observed fluctuating structures, defined as the $1 / e$ folding length for the correlation coefficient $\rho$ at time delay $\tau=0$ calculated for a set of pairs of channels with different radial separation. The correlation length estimated for discharges with positive and negative triangularity, in two different radial position, is shown in figure 6 . Larger structures are estimated for $\delta>0$ compared to $\delta<0$ plasmas in both positions. Smaller correlation lengths are usually associated with reduced local transport [28]
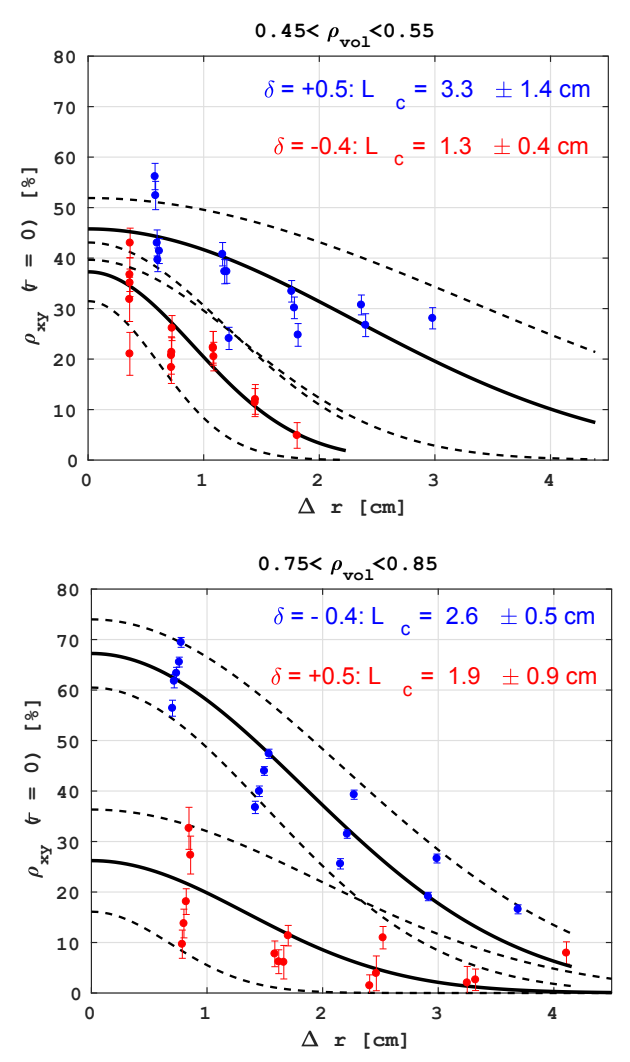

Figure 6: Correlation coefficient, at time delay $\tau=0$, for couples of channels at distance $\Delta r$, and fits for radial correlation lengths calculated for sets of six CECE channels at $0.75<\rho_{\text {vol }}<0.85$ and $0.45<$ $\rho_{\text {vol }}<0.55$. Colors online.

\section{Conclusion}

A series of discharges, with similar density profiles and plasma current, in which triangularity was varied from $\delta=0.5$ to $\delta=-0.4$ has been performed in TCV. Fluctuation measurements over the region $0.4<\rho_{\text {vol }}<$ 0.9 have been made using the newly upgraded CECE diagnostic. The data show a strong reduction in the fluctuations amplitude at the edge of these plasmas with negative triangularity. This suppression seems to penetrate up to $\rho_{v o l}=0.55$ where the triangularity at this flux surface (0.12) is already much lower than at the edge (less than $\sim 40 \%$ of the edge value).

When the fluctuation data are plotted versus the normalized scale lengths at the position of the emission volumes, a threshold behaviour is observed both on density and temperature for the positive triangularity discharges but not in the negative triangularity ones. One hypothesis is that in the latter case the threshold scale length is higher with respect to the range that has been explored in these experiments. This is in agreement with the results of nonlinear gyrokinetic flux tube simulations. These measurements are taken over 
a considerable extension of the minor radius where the scale lengths are not the only parameters varying. But due to the extreme similarity of the profiles of many other quantities and the fact that these scale lengths are supposed to constitute the drives for the observed fluctuations, these trends could show the presence of a higher threshold for fluctuations onset in negative triangularity plasmas.

Estimation of the radial correlation length has shown that it is shorter in the negative triangularity discharges than in positive triangularity cases, a condition usually associated with improved confinement. All these observations suggest that an underlying difference exists in the nature of the turbulence fluctuations in negative and positive triangularity plasmas. Further work is necessary to identify the underlying cause of this difference.

Future experiments will focus on the effects of different ratios of electron and ion temperature in plasmas with positive and negative $\delta$ to investigate the effects of a transition between TEM to ITG dominated turbulence regime on the fluctuations.

Moreover a study of the dependence of fluctuations in differently shaped plasmas at varying collisionality will be undertaken, to verify if the difference in fluctuations between positive and negative triangularity plasmas tends to decrease with increasing collisionality as does the heat flux [5]. At the same time the study of the critical gradients will continue with the exploration of a larger parameter space.

\section{Acknowledgements}

This work was performed partly in the framework of the Helmholtz Virtual Institute on Plasma Dynamical Processes and Turbulence Studies using Advanced Microwave Diagnostics. This work was also supported in part by the Swiss National Science Foundation and has been carried out within the framework of the EUROfusion Consortium and has received funding from the Euratom research and training programme 2014-2018 under grant agreement No 633053. The views and opinions expressed herein do not necessarily reflect those of the European Commission.

\section{Bibliography}

[1] S. Coda and The TCV team. Overview of the TCV tokamak program: scientific progress and facility upgrades. Nuclear Fusion, 57(10):102011, 2017.

[2] T. C. Luce. An analytic functional form for characterization and generation of axisymmetric plasma boundaries. Plasma Physics and Controlled Fusion, 55(9):095009, 2013.

[3] J.-M. Moret, S. Franke, H. Weisen, M. Anton, R. Behn, B. P. Duval, F. Hofmann, B. Joye, Y. Martin, C. Nieswand, Z. A. Pietrzyk, and W. van Toledo. Influence of Plasma Shape on Transport in the TCV
Tokamak. Physical Review Letters, 79(11):2057-2060, September 1997.

[4] Y. Camenen, A. Pochelon, A. Bottino, S. Coda, F. Ryter, O. Sauter, R. Behn, T. P. Goodman, M. A. Henderson, A Karpushov, L. Porte, and G. Zhuang. Electron heat transport in shaped TCV L-mode plasmas. Plasma Physics and Controlled Fusion, 47(11):1971, 2005.

[5] Y. Camenen, A. Pochelon, R. Behn, A. Bottino, A. Bortolon, S. Coda, A. Karpushov, O. Sauter, G. Zhuang, and the TCV team. Impact of plasma triangularity and collisionality on electron heat transport in TCV L-mode plasmas. Nuclear Fusion, 47(7):510, 2007.

[6] A. Marinoni, S. Brunner, Y. Camenen, S. Coda, J. P. Graves, X. Lapillonne, A. Pochelon, O. Sauter, and L. Villard. The effect of plasma triangularity on turbulent transport: modeling TCV experiments by linear and non-linear gyrokinetic simulations. Plasma Physics and Controlled Fusion, 51(5):055016, 2009.

[7] G. Merlo, S. Brunner, O. Sauter, Y. Camenen, T. Görler, F. Jenko, A. Marinoni, D. Told, and L. Villard. Investigating profile stiffness and critical gradients in shaped TCV discharges using local gyrokinetic simulations of turbulent transport. Plasma Physics and Controlled Fusion, 57(5):054010, 2015.

[8] Gabriele Merlo. Flux-tube and global grid-based gyrokinetic simulations of plasma microturbulence and comparisons with experimental TCV measurements. PhD thesis, 2016.

[9] O. Sauter, S. Brunner, D. Kim, G. Merlo, R. Behn, Y. Camenen, S. Coda, B. P. Duval, L. Federspiel, T. P. Goodman, A. Karpushov, A. Merle, and Tcv Team. On the non-stiffness of edge transport in L-mode tokamak plasmas. Physics of Plasmas, 21(5):055906, May 2014.

[10] Mike Kotschenreuther, G. Rewoldt, and W. M. Tang. Comparison of initial value and eigenvalue codes for kinetic toroidal plasma instabilities. Computer Physics Communications, 88(2):128-140, August 1995.

[11] F. Jenko, W. Dorland, M. Kotschenreuther, and B. N. Rogers. Electron temperature gradient driven turbulence. Physics of Plasmas, 7(5):1904-1910, April 2000.

[12] Christopher Watts. A Review of ECE Correlation Radiometry Techniques for Detection of Core Electron Temperature Fluctuations. Fusion Science and Technology, 52(2):176-192, August 2007.

[13] G. Cima. Core temperature fluctuations and related heat transport in the Texas Experimental Tokamak-Upgrade. Physics of Plasmas, 2(3):720-726, March 1995.

[14] H. J. Hartfuß, M. Häse, C. Watts, M. Hirsch, T. Geist, and the W7-AS Team. Temperature fluctuation measurements with ECE on W7-AS. Plasma Physics and Controlled Fusion, 38(12A):A227, 1996.

[15] A. E. White, L. Schmitz, W. A. Peebles, T. A. Carter, T. L. Rhodes, E. J. Doyle, P. A. Gourdain, J. C. Hillesheim, G. Wang, C. Holland, G. R. Tynan, M. E. Austin, G. R. McKee, M. W. Shafer, K. H. Burrell, J. Candy, J. C. DeBoo, R. Prater, G. M. Staebler, R. E. Waltz, and M. A. Makowski. A correlation electron cyclotron emission diagnostic and the importance of multifield fluctuation measurements for testing nonlinear gyrokinetic turbulence simulations. Review of Scientific Instruments, 79(10):103505, October 2008.

[16] C. Sung, A. E. White, J. H. Irby, R. Leccacorvi, R. Vieira, C. Y. Oi, W. A. Peebles, and X. Nguyen. Design of a correlation electron cyclotron emission diagnostic for Alcator C-Mod. Review of Scientific Instruments, 83(10):10E311, July 2012.

[17] S. J. Freethy, G. D. Conway, I. Classen, A. J. Creely, T. Happel, A. Köhn, B. Vanovac, and A. E. White. Measurement of turbulent electron temperature 
fluctuations on the ASDEX Upgrade tokamak using correlated electron cyclotron emission. Review of Scientific Instruments, 87(11):11E102, November 2016.

[18] Y. Kogi, T. Higashi, N. Tamura, H. Tsuchiya, D. Kuwahara, Y. Nagayama, A. Mase, K. Takehara, and T. Tokuzawa. Observation of electron temperature turbulence with a correlation electron cyclotron emission radiometer on LHD. Journal of Instrumentation, 11(01):C01072, 2016.

[19] M. Fontana, L. Porte, and P. Molina Cabrera. Correlation electron cyclotron emission diagnostic in TCV. Review of Scientific Instruments, 88(8):083506, August 2017.

[20] R. Behn, J. H. Rommers, R. A. Pitts, Z. A. Pietrzyk, R. Chavan, and B. Marletaz. A Thomson scattering diagnostic for measurements in the divertor region of TCV. Review of Scientific Instruments, 70(1):768-771, January 1999.

[21] Alessandro Bortolon. Plasma rotation and momentum transport studies in the TCV tokamak based on charge exchange spectroscopy measurements. 2009.

[22] T. D. Rempel, R. F. Gandy, and A. J. Wootton. Density fluctuation effects on electron cyclotron emission correlation measurements in optically gray plasmas. Review of Scientific Instruments, 65(6):2044-2048, June 1994.

[23] M. E. Austin, R. F. Ellis, and T. C. Luce. Determination of wall reflectivity for ECE frequencies in DIII-D. Article, January 1997.

[24] K. Matsuda. Ray tracing study of the electron cyclotron current drive in DIII-D using $60 \mathrm{GHz}$. IEEE Transactions on Plasma Science, 17(1):6-11, February 1989.

[25] Ronald H. Cohen. Effect of trapped electrons on current drive. The Physics of Fluids, 30(8):2442-2449, August 1987.

[26] Motoki Nakata, Masanori Nunami, Hideo Sugama, and Tomo-Hiko Watanabe. Isotope Effects on TrappedElectron-Mode Driven Turbulence and Zonal Flows in Helical and Tokamak Plasmas. Physical Review Letters, 118(16):165002, April 2017.

[27] H. Reimerdes, A. Pochelon, O. Sauter, T. P. Goodman, M. A. Henderson, and An Martynov. Effect of triangular and elongated plasma shape on the sawtooth stability. Plasma Physics and Controlled Fusion, 42(6):629, 2000.

[28] A. J. Wootton, B. A. Carreras, H. Matsumoto, K. McGuire, W. A. Peebles, Ch. P. Ritz, P. W. Terry, and S. J. Zweben. Fluctuations and anomalous transport in tokamaks. Physics of Fluids B: Plasma Physics, 2(12):2879-2903, December 1990. 